\title{
Exception to the rule?
}

DOI:

$10.1038 / \mathrm{nrm} 2279$

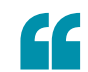

...the immortal

strand model

[...] can no

longer be

considered

a general

characteristic

of stem-cell

division.
The asymmetric segregation of chromosomes during cell division is proposed to be a characteristic of stem cells, and this 'immortal strand hypothesis' was proposed as a way in which stem cells protect themselves from accumulating mutations.

A related hypothesis is that the nucleotide analogue bromodeoxyuridine (BrdU) is selectively retained by stem cells - either because they segregate chromosomes asymmetrically or because they divide slowly. New findings now suggest that at least one type of stem cell does not conform to these ideas.

As haematopoietic stem cells (HSCs) can be highly purified using well-characterized markers, Sean Morrison and colleagues tested both the immortal strand and the BrdU retention hypotheses. They administered BrdU to adult mice for 10 days, and BrdU retention was measured after another 70 days without BrdU. According to the authors' modelling, if HSCs segregate their chromosomes asymmetrically, then only $0.6 \%$ of HSCs would retain BrdU. If HSCs segregate their chromosomes randomly, then BrdU should be lost stochastically over time, and the threshold at which BrdU can be detected by immunofluorescence would determine the measured retention. The authors predicted that few $(<20 \%)$ HSCs would retain BrdU irrespective of how chromosomes are segregated. Indeed, the authors found that $4.6 \%$ of HSCs and $0.4 \%$ of all bone marrow cells retained BrdU (only $0.08 \%$ of labelled bone marrow cells were HSCs), which demonstrates that $\mathrm{BrdU}$ retention has poor sensitivity and specificity as an HSC marker.

The immortal strand hypothesis suggests that stem cells can incorporate BrdU into DNA strands that become the 'older' strands during symmetric cell divisions, and these labelled strands are retained when stem cells resume asymmetric divisions. To test this possibility, the authors administered BrdU for 10 days to newborn mice and granulocyte colony-stimulating factor (G-CSF)/cyclophosphamide-treated mice, both of which have symmetrically dividing HSCs. Asymmetric stem-cell division was resumed and following 70 treatment-free days, only $2-6 \%$ of HSCs had retained the label and only $0.2-0.4 \%$ of BrdU-retaining bone marrow cells were HSCs. These

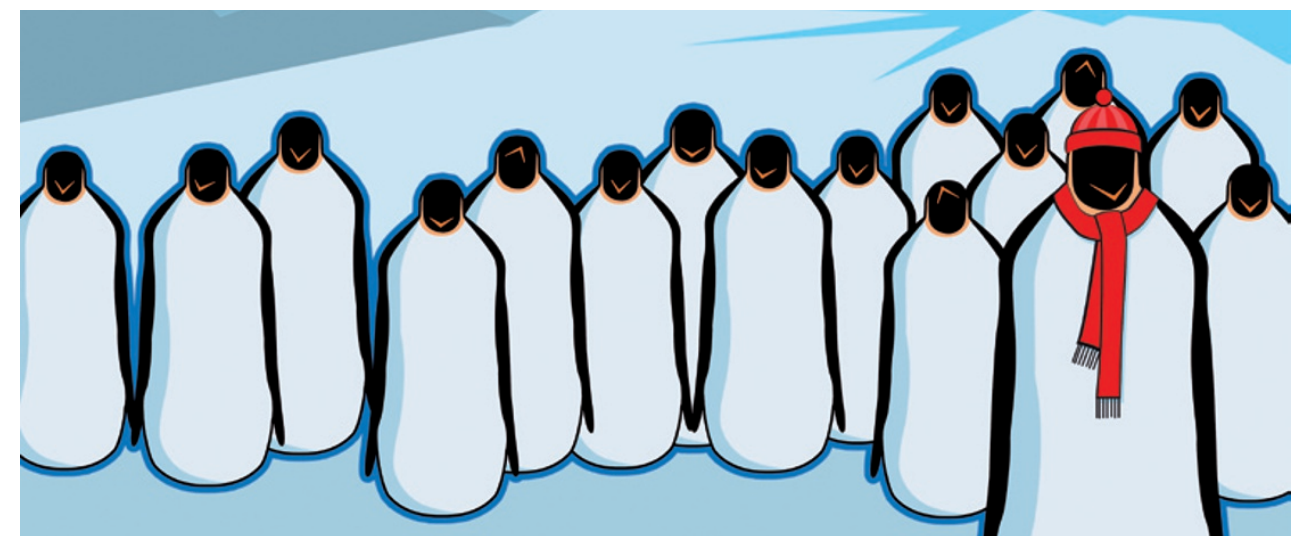

results confirmed that BrdU retention was a poor marker for HSCs and suggested random chromosome segregation.

To test the immortal strand hypothesis directly, Morrison and colleagues treated mice with one nucleotide analogue for 10 days and then with a second analogue for another 10 days. In the case of asymmetric chromosome segregation, HSCs should rarely incorporate both markers, as newly labelled chromosomes should be segregated to daughter cells. By contrast, if segregation is random, then labelled HSCs should have the same chance $(50 \%)$ as unlabelled cells of incorporating the second label, and $25 \%(50 \% \times 50 \%)$ should therefore be double labelled. Indeed, $27 \%$ of HSCs incorporated both labels, which contradicts the immortal strand hypothesis.

Their data were further corroborated by an experiment in which the authors isolated HSCs from mice treated with BrdU for 10 days and examined the division of individual HSCs in culture. They found that all colonies contained either labelled or unlabelled cells, as would be expected by random chromosome segregation.

So, HSCs appear to segregate chromosomes randomly, and BrdU retention is neither a sensitive nor a specific marker for HSCs. Although the immortal strand model, including BrdU retention, may well apply to other types of stem cells, it can no longer be considered a general characteristic of stem-cell division. Arianne Heinrichs

ORIGINAL RESEARCH PAPER Kiel, M. J. et al. Haematopoietic stem cells do not asymmetrically segregate chromosomes or retain BrdU. Nature 449, 238-242 (2007) 